\title{
Toeplitz Matrices Whose Elements Are the Coefficients of Functions with Bounded Boundary Rotation
}

\author{
V. Radhika, ${ }^{1}$ S. Sivasubramanian, ${ }^{2}$ G. Murugusundaramoorthy, ${ }^{3}$ and Jay M. Jahangiri ${ }^{4}$ \\ ${ }^{1}$ Department of Mathematics, Easwari Engineering College, Ramapuram, Chennai 600089, India \\ ${ }^{2}$ Department of Mathematics, University College of Engineering Tindivanam, Anna University, Tindivanam 604001, India \\ ${ }^{3}$ Department of Mathematics, School of Advanced Sciences, VIT University, Vellore 632 014, India \\ ${ }^{4}$ Department of Mathematical Sciences, Kent State University, Burton, OH 44021-9500, USA
}

Correspondence should be addressed to Jay M. Jahangiri; jjahangi@kent.edu

Received 3 July 2016; Accepted 3 August 2016

Academic Editor: Yan Xu

Copyright (c) 2016 V. Radhika et al. This is an open access article distributed under the Creative Commons Attribution License, which permits unrestricted use, distribution, and reproduction in any medium, provided the original work is properly cited.

Let $\mathscr{R}$ denote the family of functions $f(z)=z+\sum_{n=2}^{\infty} a_{n} z^{n}$ of bounded boundary rotation so that $\operatorname{Re}\left(f^{\prime}(z)\right)>0$ in the open unit disk $\mathbb{U}=\{z:|z|<1\}$. We obtain sharp bounds for Toeplitz determinants whose elements are the coefficients of functions $f \in \mathscr{R}$.

\section{Introduction}

Let $\mathscr{A}$ denote the class of all functions $f$ of the form

$$
f(z)=z+\sum_{n=2}^{\infty} a_{n} z^{n}
$$

which are analytic in the open unit disk $\mathbb{U}=\{z:|z|<1\}$ and let $\mathcal{S}$ denote the subclass of $\mathscr{A}$ consisting of univalent functions. Obviously, for functions $f \in \mathcal{S}$, we must have $f^{\prime} \neq$ 0 in $\mathbb{U}$. For $f \in \mathcal{S}$ we consider the family $\mathscr{R}$ of functions of bounded boundary rotation so that $\operatorname{Re}\left(f^{\prime}(z)\right)>0$ in $\mathbb{U}$. The family $\mathscr{R}$ is properly contained in the class of close-to-convex functions (e.g., see Brannan [1], Pinchuk [2], or Duren [3] pp. 269-271.)

Toeplitz matrices are one of the well-studied classes of structured matrices. They arise in all branches of pure and applied mathematics, statistics and probability, image processing, quantum mechanics, queueing networks, signal processing, and time series analysis, to name a few (e.g., see Ye and Lim [4]). Toeplitz matrices have some of the most attractive computational properties and are amenable to a wide range of disparate algorithms and determinant computations. Here we consider the symmetric Toeplitz determinant

$$
T_{q}(n)=\left|\begin{array}{cccc}
a_{n} & a_{n+1} & \cdots & a_{n+q-1} \\
a_{n+1} & \cdots & & \vdots \\
\vdots & & & \\
a_{n+q-1} & \cdots & & a_{n}
\end{array}\right|
$$

and obtain sharp bounds for the coefficient body $\left|T_{q}(n)\right| ; q=$ 2,$3 ; n=1,2,3$, where the entries of $T_{q}(n)$ are the coefficients of functions $f$ of form (1) that are in the family $\mathscr{R}$ of functions of bounded boundary rotation. As far as we are concerned, the results presented here are new and noble and the only prior compatible result is published by Thomas and Halim [5] for the classes of starlike and close-to-convex functions. It is worth noticing that the bounds presented here are much finer than those presented in [5].

\section{Main Results}

We note that, for the functions $f$ of form (1) that are in the family $\mathscr{R}$ of functions of bounded boundary rotation, we can 
write $f^{\prime}(z)=h(z)$, where $h \in \mathscr{P}$, the class of positive real part function satisfying $\operatorname{Re}(h(z))>0$ for $z \in \mathbb{U}$ and $h$ is of the form

$$
h(z)=1+\sum_{n=1}^{\infty} c_{n} z^{n}
$$

We shall state the following result [6], to prove our main theorems.

Lemma 1. Let $h(z)=1+\sum_{n=1}^{\infty} c_{n} z^{n} \in \mathscr{P}$. Then for some complex valued $x$ with $|x| \leq 1$ and some complex valued $\zeta$ with $|\zeta| \leq 1$ we have

$$
\begin{aligned}
2 c_{2}= & c_{1}^{2}+x\left(4-c_{1}^{2}\right) \\
4 c_{3}= & c_{1}^{3}+2\left(4-c_{1}^{2}\right) c_{1} x-c_{1}\left(4-c_{1}^{2}\right) x^{2} \\
& +2\left(4-c_{1}^{2}\right)\left(1-|x|^{2}\right) \zeta .
\end{aligned}
$$

In our first theorem we obtain a sharp bound for the coefficient body $\left|T_{2}(2)\right|$.

Theorem 2. Let $f \in \mathscr{R}$ be given by (1). Then we have the sharp bound

$$
\left|T_{2}(2)\right|=\left|a_{3}^{2}-a_{2}^{2}\right| \leq \frac{5}{9} .
$$

Proof. First note that by equating the corresponding coefficients of $f^{\prime}(z)=h(z)$, we obtain

$$
\begin{aligned}
& a_{2}=\frac{c_{1}}{2} \\
& a_{3}=\frac{c_{2}}{3} \\
& a_{4}=\frac{c_{3}}{4} .
\end{aligned}
$$

Now by (2), (6), and (7) we have

$$
\left|T_{2}(2)\right|=\left|\left(\left|\begin{array}{ll}
a_{2} & a_{3} \\
a_{3} & a_{2}
\end{array}\right|\right)\right|=\left|a_{2}^{2}-a_{3}^{2}\right|=\left|\frac{c_{1}^{2}}{4}-\frac{c_{2}^{2}}{9}\right| .
$$

Making use of Lemma 1 to express $c_{2}$ in terms of $c_{1}$, we obtain

$$
\left|a_{3}^{2}-a_{2}^{2}\right|=\left|\frac{c_{1}^{2}}{4}-\frac{\left[c_{1}^{2}+x\left(4-c_{1}^{2}\right)\right]^{2}}{36}\right| .
$$

Without loss of generality, let $0 \leq c_{1}=c \leq 2$. Applying triangle inequality, we get

$$
\begin{aligned}
& \left|a_{3}^{2}-a_{2}^{2}\right| \\
& \leq \frac{1}{36}\left[\left(4-c^{2}\right)^{2}|x|^{2}+2 c^{2}\left(4-c^{2}\right)|x|+c^{2}\left(9-c^{2}\right)\right] \\
& =: \Phi(c ;|x|) .
\end{aligned}
$$

Differentiating $\Phi(c ;|x|)$ with respect to $c$ we obtain

$$
\begin{aligned}
& \frac{\partial \Phi(c ;|x|)}{\partial c} \\
& =\frac{c}{18}\left[2\left(|x|^{2}-2|x|-1\right) c^{2}-\left(8|x|^{2}-8|x|-9\right)\right] .
\end{aligned}
$$

Setting $\partial \Phi(c ;|x|) / \partial c=0$ leads to either $c=0$ or

$$
c^{2}=\frac{9+8|x|-8|x|^{2}}{1+2|x|-|x|^{2}} .
$$

But $\left(9+8|x|-8|x|^{2}\right) /\left(1+2|x|-|x|^{2}\right)>4$ for $|x| \leq 1$. Therefore the maximum of $\left|a_{3}^{2}-a_{2}^{2}\right|$ occurs either at $c=0$ or $c=2$.

For $c=0$ we obtain $a_{2}=0$ and $a_{3}=2 x / 3$ which implies $\left|a_{3}^{2}-a_{2}^{2}\right|=\left|(2 x / 3)^{2}\right| \leq 4 / 9$.

For $c=2$ we obtain $a_{2}=1$ and $a_{3}=2 / 3$ which implies $\left|a_{3}^{2}-a_{2}^{2}\right|=\left|1-(2 / 3)^{2}\right| \leq 5 / 9$.

This bound is sharp and the extremal function is given by $f^{\prime}(z)=(1+z) /(1-z)$.

We remark that the sharp bound $\left|a_{3}^{2}-a_{2}^{2}\right| \leq 5 / 9$ given by Theorem 2 is much finer than $\left|a_{3}^{2}-a_{2}^{2}\right| \leq 5$ that was obtained by Thomas and Halim [5] for the class of functions of form (1) that are close-to-convex in $\mathbb{U}$. $\left|T_{2}(3)\right|$.

Next, we determine a sharp bound for the coefficient body

Theorem 3. Let $f \in \mathscr{R}$ be given by (1). Then we have the sharp bound

$$
\left|T_{2}(3)\right|=\left|a_{4}^{2}-a_{3}^{2}\right| \leq \frac{4}{9} .
$$

Proof. Note that, by (2), (7), and (8), we have

$$
\left|T_{2}(3)\right|=\left|\left(\left|\begin{array}{ll}
a_{3} & a_{4} \\
a_{4} & a_{3}
\end{array}\right|\right)\right|=\left|a_{4}^{2}-a_{3}^{2}\right|=\left|\frac{c_{3}^{2}}{16}-\frac{c_{2}^{2}}{9}\right| .
$$

Making use of Lemma 1 and triangle inequality, we obtain

$$
\begin{aligned}
& \left|a_{4}^{2}-a_{3}^{2}\right| \leq\left(\frac{M^{2} c^{2}}{256}+\frac{M^{2}}{64}-\frac{M^{2} c}{64}\right)|x|^{4} \\
& +\left(\frac{M^{2} c^{2}}{64}-\frac{M^{2} c}{32}\right)|x|^{3} \\
& +\left(\frac{M^{2} c^{2}}{64}-\frac{M^{2}}{288}+\frac{M c^{4}}{128}-\frac{M c^{3}}{64}+\frac{M^{2} c}{64}+\frac{M c^{2}}{18}\right) \\
& \cdot|x|^{2}+\left(\frac{M c^{4}}{64}+\frac{M^{2} c}{32}\right)|x|+\frac{M c^{3}}{64}+\frac{M^{2}}{64}+\frac{c^{4}}{36} \\
& -\frac{c^{6}}{256}:=\Phi(c,|x|),
\end{aligned}
$$

where, without loss of generality, we let $0 \leq c_{1}=c \leq 2$ and $M=4-c^{2}$.

Differentiating and using a simple calculus shows that $\partial \Phi(c,|x|) / \partial|x| \geq 0$ for $|x| \in[0,1]$ and fixed $c \in[0,2]$. 
It follows that $\Phi(c,|x|)$ is an increasing function of $|x|$. So $\Phi(c,|x|) \leq \Phi(c, 1)$. Upon letting $|x|=1$, a simple algebraic manipulation yields

$$
\left|a_{4}^{2}-a_{3}^{2}\right| \leq \frac{64-9 c^{2}}{144} \leq \frac{4}{9} .
$$

This bound is sharp and the extremal function is given by $f^{\prime}(z)=\left(1+z^{2}\right) /\left(1-z^{2}\right)$.

No bounds for $\left|a_{4}^{2}-a_{3}^{2}\right|$ was obtained by Thomas and Halim [5] for the class of functions of form (1) that are closeto-convex in $\mathbb{U}$. In our next theorem we determine a sharp bound for the coefficient body $\left|T_{3}(1)\right|$.

Theorem 4. Let $f \in \mathscr{R}$ be given by (1). Then we have the sharp bound

$$
\left|T_{3}(1)\right|=\left|\left(\left|\begin{array}{lll}
1 & a_{2} & a_{3} \\
a_{2} & 1 & a_{2} \\
a_{3} & a_{2} & 1
\end{array}\right|\right)\right| \leq \frac{13}{9} .
$$

Proof. Expanding the determinant $T_{3}(1)$ and letting $M=4-$ $c^{2}$ we obtain

$$
\begin{aligned}
\left|T_{3}(1)\right| & =\left|1+2 a_{2}^{2}\left(a_{3}-1\right)-a_{3}^{2}\right| \\
& =\left|1+\frac{c_{1}^{2}}{2}\left(\frac{c_{2}}{3}-1\right)-\frac{c_{2}^{2}}{9}\right| \\
& =\left|1+\frac{1}{18} c_{1}^{4}-\frac{1}{2} c_{1}^{2}+\frac{1}{36} c_{1}^{2} x M-\frac{1}{36} x^{2} M^{2}\right| .
\end{aligned}
$$

As before, without loss of generality, we assume that $c_{1}=c$, where $0 \leq c \leq 2$. Then, by using the triangle inequality and the fact that $|x| \leq 1$, we obtain

$$
\begin{gathered}
\left|T_{3}(1)\right| \leq\left|1+\frac{1}{18} c^{4}-\frac{1}{2} c^{2}\right|+\frac{1}{36} c^{2}\left(4-c^{2}\right) \\
+\frac{1}{36}\left(4-c^{2}\right)^{2}:=\Psi(c) .
\end{gathered}
$$

Considering the modulus as positive, we get

$$
\Psi(c)=\frac{1}{18}\left[c^{4}-11 c^{2}+26\right] .
$$

One can apply an elementary calculus to show that $\Psi(c)$ attains its maximum value of $13 / 9$ on $[0,2]$ when $c=0$. Similarly, considering the modulus as negative, we obtain

$$
\Psi(c)=\frac{1}{18}\left[-c^{4}+7 c^{2}-10\right]
$$

Again, using an elementary calculus argument shows that this expression has a maximum value of $1 / 8$ on $[0,2]$ when $c=$ $\sqrt{7 / 2}$. The sharp bound $\left|T_{3}(1)\right|=13 / 9$ is achieved for $c_{1}=0$ and $c_{2}=2 i$.

We remark that the sharp bound $\left|T_{3}(1)\right| \leq 13 / 9$ given by Theorem 4 is much finer than $\left|T_{3}(1)\right| \leq 8$ obtained by Thomas and Halim [5] for the class of functions of form (1) that are close-to-convex in $\mathbb{U}$. Finally, an upper bound for the coefficient body $\left|T_{3}(2)\right|$ is presented in the following.
Theorem 5. Let $f \in \mathscr{R}$ be given by (1). Then we have the upper bound

$$
\left|T_{3}(2)\right|=\left|\left(\left|\begin{array}{lll}
a_{2} & a_{3} & a_{4} \\
a_{3} & a_{2} & a_{3} \\
a_{4} & a_{3} & a_{2}
\end{array}\right|\right)\right| \leq \frac{4}{9} .
$$

Proof. Write

$$
\left|T_{3}(2)\right|=\left|\left(a_{2}-a_{4}\right)\left(a_{2}^{2}-2 a_{3}^{2}+a_{2} a_{4}\right)\right| .
$$

Using the same techniques as above, one can obtain with simple computations that $\left|a_{2}-a_{4}\right| \leq 1 / 2$. Thus we need to show that $\left|a_{2}^{2}-2 a_{3}^{2}+a_{2} a_{4}\right| \leq 8 / 9$. In view of (6), (7), and (8), a simple computation leads to

$$
\left|a_{2}^{2}-2 a_{3}^{2}+a_{2} a_{4}\right|=\left|\frac{c_{1}^{2}}{4}-\frac{2 c_{2}^{2}}{9}+\frac{c_{1} c_{3}}{8}\right| .
$$

Expressing $c_{2}$ and $c_{3}$ in terms of $c_{1}$ as earlier and using Lemma 1 with $M=4-c_{1}^{2}$ and $N=\left(1-|x|^{2}\right) \zeta$, we obtain

$$
\begin{gathered}
\left|a_{2}^{2}-2 a_{3}^{2}+a_{2} a_{4}\right|=\mid \frac{c_{1}^{2}}{4}-\frac{c_{1}^{4}}{18}-\frac{x^{2} M^{2}}{18}-\frac{7}{144} c_{1}^{2} M x \\
+\frac{1}{32} c_{1}^{4}-\frac{1}{32} c_{1}^{2} M x^{2}+\frac{1}{16} c_{1} x N \mid .
\end{gathered}
$$

Applying the triangle inequality and assuming that $0 \leq c_{1}=$ $c \leq 2$, we obtain

$$
\begin{aligned}
\left|a_{2}^{2}-2 a_{3}^{2}+a_{2} a_{4}\right| \leq & \left|\frac{c^{2}}{4}-\frac{7}{288} c^{4}\right|+\frac{1}{18}|x|^{2}\left(4-c^{2}\right)^{2} \\
& +\frac{7}{144} c^{2}\left(4-c^{2}\right)|x| \\
& +\frac{1}{32} c^{2}\left(4-c^{2}\right)|x|^{2} \\
& +\frac{1}{16} c\left(4-c^{2}\right)\left(1-|x|^{2}\right) \\
:= & \mu(c,|x|) .
\end{aligned}
$$

We need to find the maximum value of $\mu(c,|x|)$ on $[0,2] \times$ $[0,1]$. First, assume that there is a maximum at anterior point $\left(c,\left|x_{0}\right|\right)$ of $[0,2] \times[0,1]$. Then differentiating $\mu\left(c_{0},|x|\right)$ with respect to $|x|$ and equaling it to 0 would imply that $c_{0}=2$, which is a contradiction. Thus to find the maximum of $\mu(c,|x|)$, we need only to consider the end points of $[0,2] \times$ $[0,1]$.

When $c=0, \mu(0,|x|)=(16 / 18)|x|^{2} \leq 8 / 9$.

When $c=2, \mu(2,|x|)=11 / 18$.

When $|x|=0, \mu(c, 0)=\left|c^{2} / 4-(7 / 288) c^{4}\right|+(1 / 16) c\left(4-c^{2}\right)$, which has maximum value $11 / 18$ on $[0,2]$.

When $|x|=1, \mu(c, 1)=\left|c^{2} / 4-(7 / 288) c^{4}\right|+(1 / 18)(4-$ $\left.c^{2}\right)^{2}+(7 / 144) c^{2}\left(4-c^{2}\right)+(1 / 32) c^{2}\left(4-c^{2}\right)$, which has maximum value $8 / 9$ on $[0,2]$.

No bounds for $\left|T_{3}(2)\right|$ were obtained by Thomas and Halim [5] for the class of functions of form (1) that are closeto-convex in $\mathbb{U}$. 


\section{Competing Interests}

The authors declare that there are no competing interests regarding the publication of this paper.

\section{Acknowledgments}

The work of the second author is supported by a grant from Department of Science and Technology, Government of India vide ref: SR/FTP/MS-022/2012 under fast track scheme.

\section{References}

[1] D. A. Brannan, "On functions of bounded boundary rotation. I," Proceedings of the Edinburgh Mathematical Society, vol. 16, no. 2, pp. 339-347, 1968.

[2] B. Pinchuk, "A variational method for functions of bounded boundary rotation," Transactions of the American Mathematical Society, vol. 138, pp. 107-113, 1969.

[3] P. L. Duren, Univalent Functions, vol. 259 of Grundlehren der Mathematischen Wissenschaften, Springer, New York, NY, USA, 1983.

[4] K. Ye and L.-H. Lim, "Every matrix is a product of Toeplitz matrices," Foundations of Computational Mathematics, vol. 16, no. 3, pp. 577-598, 2016.

[5] D. K. Thomas and S. A. Halim, "Toeplitz matrices whose elements are the coefficients of starlike and close-to-convex functions," Bulletin of the Malaysian Mathematical Sciences Society, 2016.

[6] R. J. Libera and E. J. Zloktiewicz, "Coefficient bounds for the inverse of a function with derivative in P," Proceedings of the American Mathematical Society, vol. 87, no. 2, pp. 251-257, 1983. 


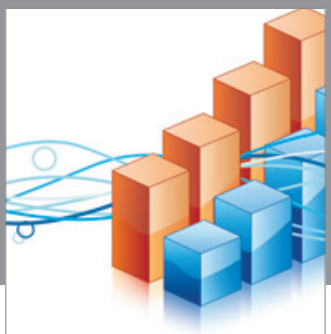

Advances in

Operations Research

vatem alat4

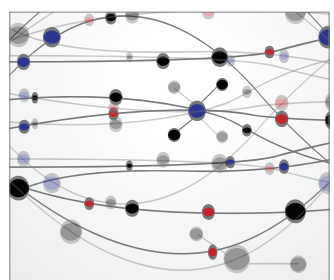

\section{The Scientific} World Journal
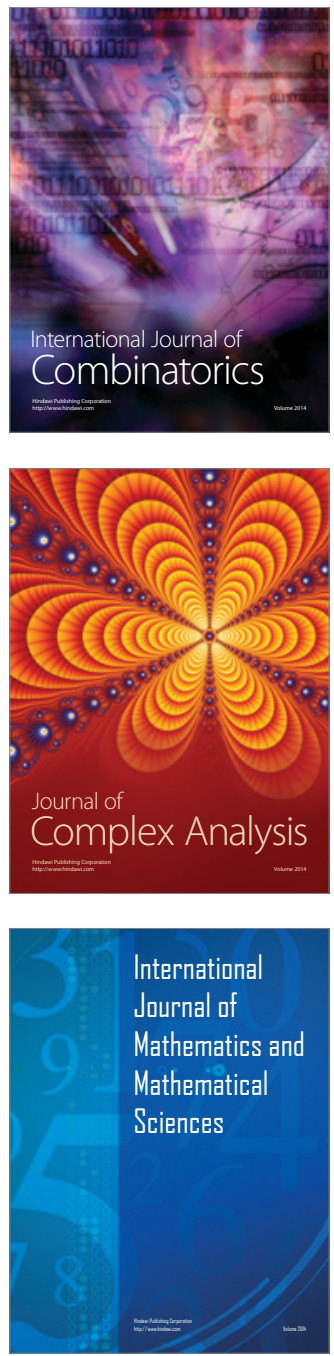
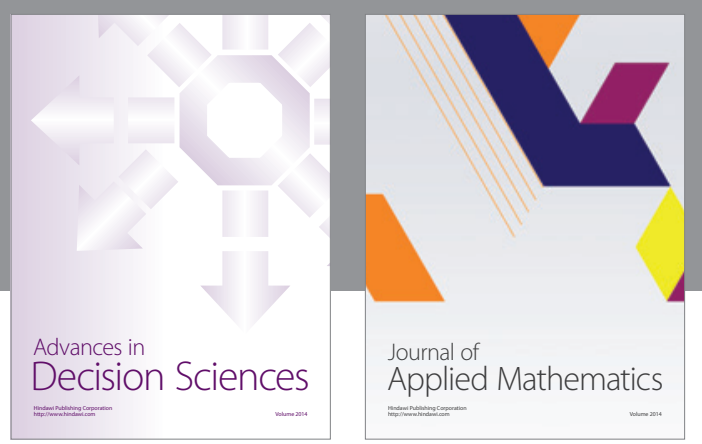

Algebra

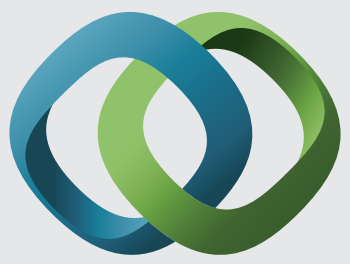

\section{Hindawi}

Submit your manuscripts at

http://www.hindawi.com
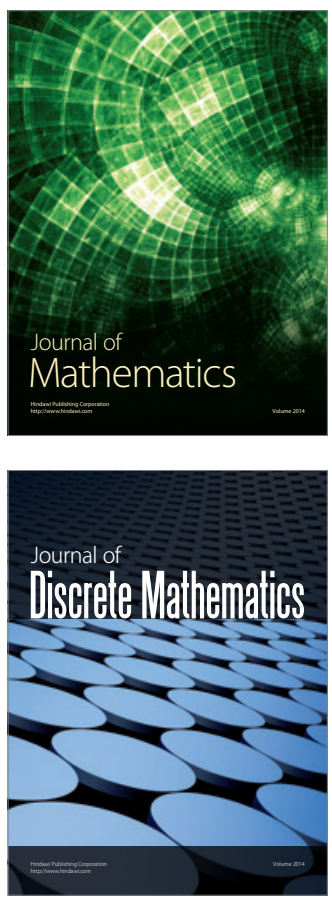

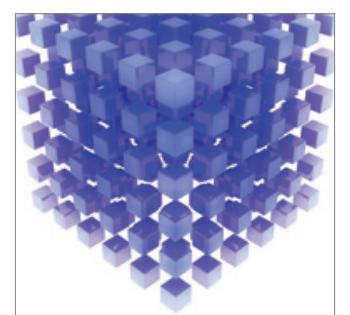

Mathematical Problems in Engineering
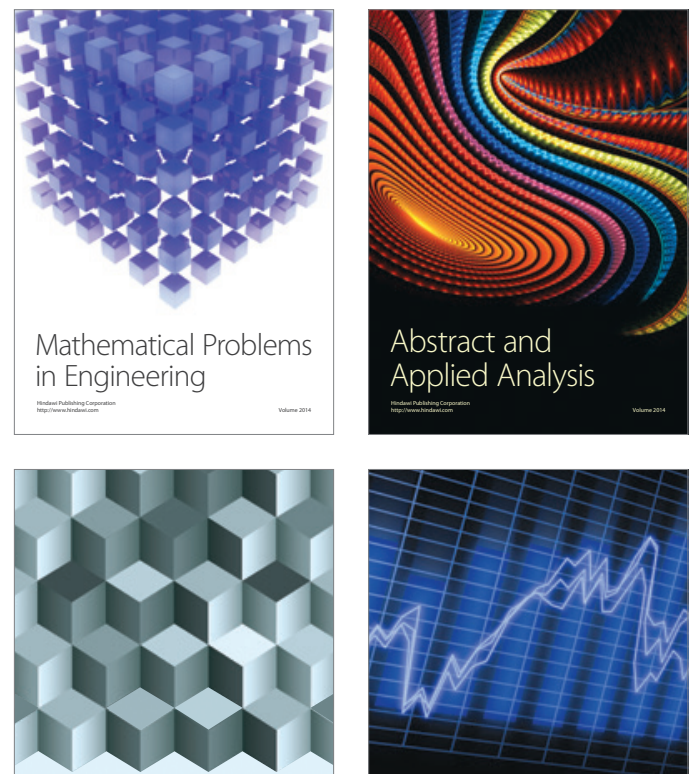

Journal of

Function Spaces

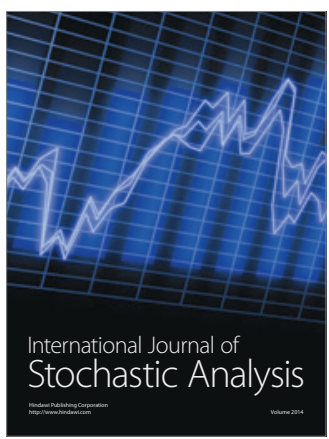

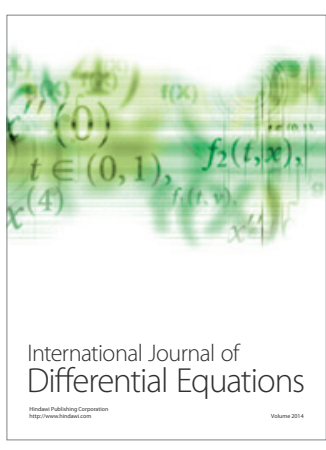
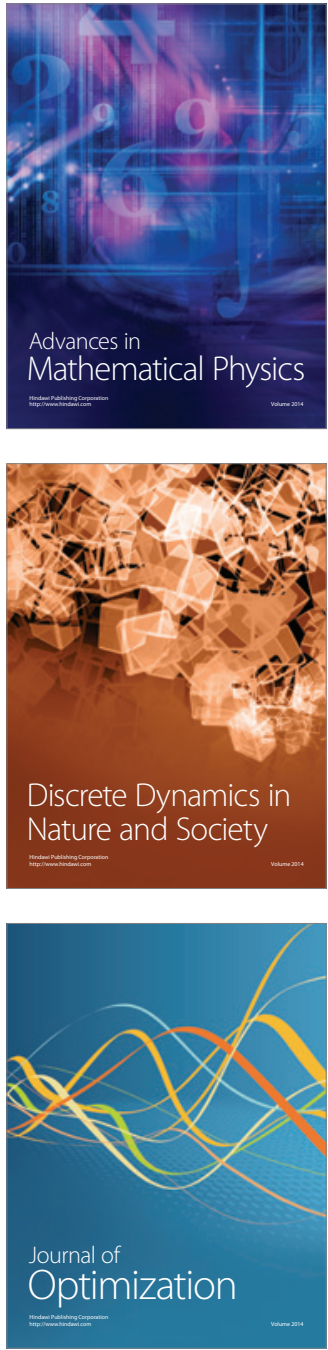